\title{
Resistivity Inversion for High-Resolution Dual Induction Logging Device
}

\author{
Jianhua Zhang \\ Science College, Xi'an Shiyou University, Xi'an, 710065, China \\ e-mail: jhzhang@xsyu.edu.cn
}

Keywords: inversion; HRDI logging; resistivity; invasion

\begin{abstract}
A fast and efficient inversion algorithm was suggested for high-resolution dual laterolog (HRDI) tool. The algorithm can yield reliable estimates for the depth of invasion zone and trueformation resistivity simultaneously. Both the behaviour of HRDL measurements and inversion were studied for various bed thickness from $0.4 \mathrm{~m}$ to $4 \mathrm{~m}$. The present results indicated that the HRDI tool improved the vertical resolution obviously, but the its measurements deviate the true value of formation due to the invasion and shoulder effect. These environment influences can be corrected from the inversion process, thus the original formation parameters can be obtained. A site application of the present inversion algorithm gave the distribution of the true-formation resistivity in a real formation scale. It is helpful for log analysts to evaluate the reservoirs.
\end{abstract}

\section{Introduction}

During oil exploration, the saturation of hydrocarbon is an important parameter to evaluate a reservoir. But it can not be measured directly, it is usually determined from the measurement of other parameters, such as formation resistivity. The formation resistivity can be measured using well logging devices. The common tools to measure the formation resistivity include induction devices and lateral devices [1]. The traditional dual-induction tool can provide two responses with medium and deep detective depth simultaneously, but it has poor vertical resolution so that the dual-induction measurement can not distinguish thin reservoirs. In order to improve the properties of dual induction log, Halliburton company produced a high-resolution dual induction (HRDI) logging tool [2]. It has higher vertical resolution, $0.6 \mathrm{~m}$, both for deep and medium induction array; and the radial investigation depth reach $2.3 \mathrm{~m}$ and $1 \mathrm{~m}$ for deep and medium induction array respectively.

However, the environmental effects such as borehole, mud, invasion and shoulder beds, influence the resistivity measurements. The logging responses will usually deviate from the value of true-formation resistivity. In order to obtain the true-formation resistivity from logging data, inversion techniques is used during logging interpretation. The resistivity inversion is aimed at the elimination of each environmental influence as far as possible and determination the true-formation resistivity.

In the present study, an efficient and fast inversion algorithm was suggested to inverse the trueformation resistivity and invasion depth simultaneously. The inversion algorithm was used to the inversion of both synthetic data and the field data. The present results are helpful for log analysts to obtain the invasion information and to determine the true-formation resistivity, then to estimate the initial saturation of hydrocarbon.

\section{Response of high-resolution dual induction}

After a permeable and porous formation was opened, the drilling-mud filtrate pours into the formation radially and displaces the native fluids in porous volumes under the pressure difference between borehole and formation. Thus, an invasion zone was formed in the adjacency of well bore. It will influence the measurement of well logging significantly. A negative result is that the logging responses will deviate from the true-formation resistivity, furthermore, the errors of saturation estimation and reservoir evaluation would be introduced. The invasion process affects the readings of HRDI measurements as well. 
The structure of a HRDI coil array is the same as a traditional dual induction device [2]. The deep detective array is comprised of three pairs of transmitters and receivers. Whereas the medium detective array is consisted of three transmitter coils and five receiver coils. A signal processing method [3] was used to eliminate shoulder effects and the skin effect. The logging sonde is centered in the borehole and the formation was divided into $L$ layers in vertical direction. In radial direction, the present formation model was two-dimensional axisymmetric. It included borehole with radius $r_{h}$ and mud resistivity $R_{m}$, invaded zone with depth $r_{i}$ and resistivity $R_{x o}$, formation with thickness $H$ and true-formation resistivity $R_{t}$.

Fig. 1 show the HDRI responses for various beds with different thickness, 0.4, 0.6, 1.0, 1.5, 2.0, 2.5, 3.0, 4.0 and $5.0 \mathrm{~m}$, in a synthetic formation model with true-formation resistivity $R_{t}=30 \Omega \mathrm{m}$. The beds were separated by shoulder with thickness $5 \mathrm{~m}$ and resistivity $2.0 \Omega \mathrm{m}$. The vertical direction in Fig.1 denotes the depth from the surface of ground. The horizontal direction in Fig. 1 denotes the measurement results of a HRDI device, or apparent resistivity $R_{a}$. The deep and medium data of HRDI measurements, $R_{I D}$ and $R_{I M}$, were draw in Fig.1 using heavy line and thin line respectively.

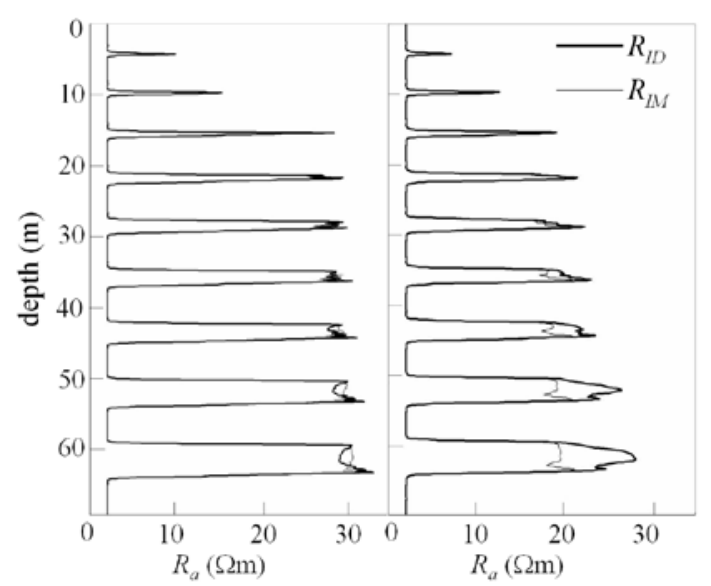

(a)

(b)

Figure 1 HDRI responses for various beds with different thickness: (a) without invasion; (b) invasion occurrence

In Fig.1a, the curves were the apparent responses $R_{a}$ without invasion, so both $R_{I D}$ and $R_{I M}$ have the same value. In Fig.2b, the logging curves of HRDI corresponded the case that invasion occurred with depth $r_{i}=0.4157 \mathrm{~m}$ and resistivity $R_{x o}=8 \Omega \mathrm{m}$. Since the shallow detective depth, the medium responses of a HRDI device obtained less reading, especially for thick bed. The deep reading of a HRDI device was controlled by original formation far away from the borehole; hence, it reflected the true-resistivity of formation much more. The present calculation results indicated that the measurement of a HRDI tool can reflect the resistivity for a thick bed. If the thickness is less than 1.0 meter, the effects of shoulder and invasion become obvious, so that the apparent resistivity will deviate the true-formation resistivity, but the HRDI measurement can still recorder the information of a thin bed, as shown in Fig.1. Comparing with traditional dual induction log tools, the HRDI tool modified the vertical resolution obviously.

\section{Inversion Algorithm}

To eliminate the negative influence of invasion, the inversion technique was usually used to recovery the true-resistivity of formation. For this purpose, the invasion zone was described by invasion depth, which denotes the distance of invasion front from the borehole, and resistivity of invaded zone. Thus, three parameters, invasion depth $r_{\mathrm{i}}$, invaded zone resistivity $R_{x o}$ and trueformation resistivity $R_{t}$, are needed to be determined from inversion technique. For the logging operation in oil fields, the HRDI usually measured with a lateral 8 (LL8) device, which recorded the information in the vicinity of borehole [1]. Therefore, the measurement result of the LL8 array can be reasonable regarded as the resistivity of invaded zone, $R_{x o}$. A high-resolution dual-induction tool 
provided two log curves with medium and deep detective depth. They were used to inverse two parameters, invasion depth $r_{\mathrm{i}}$ and true-formation resistivity $R_{t}$ from inversion method

According to the damping least square method [4-6], the logging data of HRDI tool were fit from the forward model with two unknown parameters, $r_{\mathrm{i}}$ and $R_{t}$. The logging responses satisfied following equation:

$$
R_{n}=F_{n}\left(R_{t}, r_{i}, S_{n}\right) \quad n=1,2
$$

where $R_{n}$ is logging data. $R_{1}=R_{I D}$ and $R_{2}=R_{I M}$ mean deep and medium apparent resistivities of a HRDI tool respectively. $R_{t}$ and $r_{i}$ are the inversed parameters. $S_{n}$ means other parameters in the forward model. Equation (1) is a system of nonlinear equation. If the initial guesses of the model were given, it can be linearized according to Taylar progression:

$$
R_{n}=R_{n}^{0}+\frac{\partial F_{n}}{\partial R_{t}} \delta R_{t}+\frac{\partial F_{n}}{\partial r_{i}} \delta r_{i} \quad(n=1,2)
$$

This equation can be rewritten in a matrix

$$
\begin{aligned}
& \vec{\varepsilon}=\vec{R}-\vec{R}^{0}=J \delta \vec{P} \\
& \vec{R}=\left(R_{I D}, R_{I M}\right)^{T} \\
& \vec{R}^{0}=\left(R_{I D}^{\prime}, R_{I M}^{\prime}\right)^{T} \\
& \vec{P}=\left(R_{t}, r_{i}\right)^{T}
\end{aligned}
$$

Where, $\vec{R}$ is logging data (measured resistivity or apparent responses)) vector. $\vec{R}^{0}$ denotes the guessed data vector in present forward model. The difference between them, $\varepsilon$, is the vector of the difference between logging data and guessed data. $\vec{P}$ is the inversed parameters vector and $J$ is Jacobi matrix. Equation (2) is a linear equation system related to modified step $\delta \vec{P}$. Its damping least square solution is

$$
\delta \vec{P}=\left(J^{T} J+\eta I_{0}\right)^{-1} J^{T} \vec{\varepsilon}
$$

Where $\eta$ is damping factor and $I_{0}$ is a unit matrix. The forward model was linearized at initial value $P_{0}$; then, the increment $\delta \vec{P}$ was obtained by damping least square method. New model parameters $\vec{P}^{\prime}$ is generated from the following formula

$$
\vec{P}^{\prime}=\vec{P}_{0}+\delta \vec{P}
$$

This new parameter was input into forward model and new guess data $\vec{R}^{\prime}$ was generated. Thus, new difference vector

$$
\vec{\varepsilon}=\vec{R}-\vec{R}^{\prime}
$$

and new Jacobi matrix $J$ were generated. Then the new parameter vector increment $\delta \vec{P}$ was obtained from these results. Thus, a new iteration was informed.

Equations (2) (5) were repeated until the expectant results that satisfy iteration error were reached through iteration process.

\section{Synthetic Data Inversion}

The present inversion algorithm for HRDI logging device was checked and used to a synthetic formation model with true-formation resistivity $R_{t}=5.0 \Omega \mathrm{m}$, mud resistivity $R_{m}=1.0 \Omega \mathrm{m}$, borehole radius $r_{h}=0.1016 \mathrm{~m}$, which corresponds to the diameter 8 inch for a borehole. The resistivity of invasion was set to be $R_{x o}=15 \Omega \mathrm{m}$ and the depth of invasion zone was $0.4 \mathrm{~m}$. The resistivities of shoulder bed $R_{S}=2.0 \Omega \mathrm{m}$. The reservoir thickness $H$ was chosen to be $0.4,0.6,1.0,1.5,2.0,3.0$ and $4.0 \mathrm{~m}$ respectively in order to address the vertical resolution of a HRDI tool.

The logging curves and the present inversion results for this synthetic model were shown in Fig.2. The curves in Fig.2(a) were the apparent responses $R_{a}$ of both $R_{I D}$ (the heavy line) and $R_{I M}$ (the thin line). Like the calculation results in Fig.2, HDRI readings can distinguish the thick bed effectively. For the present synthetic model, even the thickness reduced to $0.4 \mathrm{~m}$, the apparent resistivity of both $R_{I D}$ and $R_{I M}$ have certain values. 
Different from the case in Fig.1, the resistivity of invasion zone in the formation model of Fig.2 was greater than the true-resistivity of formation, hence the medium induction response was greater than the readings from the deep induction array, as shown in Fig.2(a).

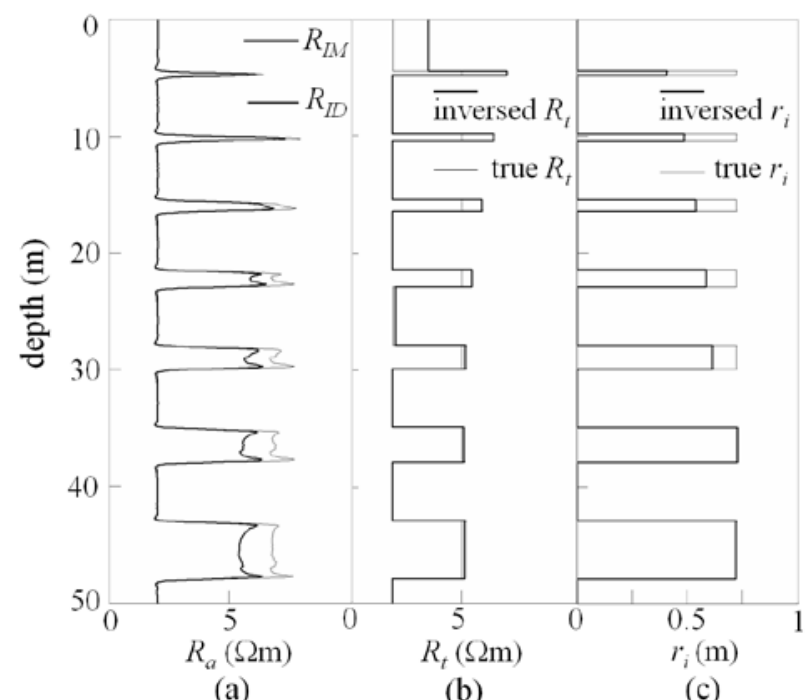

Figure 2 The present calculation results for a synthetic model:

(a) HRDI logging curves; (b) inversed true-formation resistivity;

(c) inversed invasion depth

In the present HDRI calculation in Fig.2a, the logging data deviated strong from the trueformation resistivity, $R_{t}=5.0 \Omega \mathrm{m}$, as shown in the thin line in Fig.2b, especially for the mediuminduction array in the thin beds. Due to the high-resistivity invasion zone, $R_{x o}=15 \Omega \mathrm{m}$ in the present synthetic formation model, the apparent resistivities of HDRI logging tool will greater than the trueformation resistivity usually. Invasion plays an important role in well logging and affects on the logging data strongly.

The HDRI logging data illustrated in Fig.2a were input to the present inversion model. Before inversion iterations, the formation was layered from the deep logging curves of HRDI device. The inversion iterations start with the initial guess values input. In Fig.2(b), the inversed true-formation resistivity was drawn in heavy line and true value of the formation resistivity was illustrated using the thin line. The present inversion results were drawn in Fig.2c. They indicated that for a thick bed $(H>2 \mathrm{~m})$, the inversed $R_{t}$ agreed with the true-formation resistivity. Whereas for a thin bed $(H<1 \mathrm{~m})$, the shoulder with low resistivity, $R_{s}=2.0 \Omega \mathrm{m}$, will effect the logging and inversion strongly, so that the inversion error can not be neglected.

The present inversion of HDRI logging data can also provide the invasion depth simultaneously. Fig.2c shown the inversed invasion depth $r_{i}$ (heavy line) and the true invasion depth (thin line). They were in good agreement for the present formation model for the thick beds $(H>2 \mathrm{~m})$. For the same reason, the shoulders have strong influence on the measurements of thin layers, so that the inversed invasion depths $r_{i}$ of thin layers deviated from the true values, as shown in Fig.2c.

The calculation results for the synthetic model indicated that the present inversion algorithm was efficient and reliable to obtain true-formation resistivity and invasion depth from the logging data of HDRI tool, especially for the cases of bed thickness $H>2 \mathrm{~m}$.

\section{Field Application}

In an oil field located at Western China, the high-resolution induction tool was used during well logging. Meanwhile, the well was logged using other resistivity logging device such as lateralog 8 (LL8) device simultaneously. Figure 4a was the logging curves of HRDI (the heavy line for $R_{I D}$ and the thin line for $R_{I M}$ respectively) and LL8 devices (the dash line) for a well. The horizontal axis was the apparent resistivity of logging devices and the vertical axis direction was the depth from 1908 1936m in the formation. 
Since the lateral 8 (LL8) device recorded the information in the vicinity of borehole, the measurement results of the LL8 array were input the present inversion model as the resistivity of invaded zone, $R_{x o}$. Then the HRDI measurement data in the interval 1908 1936m in Fig. 3(a) were input into the present inversion iterations with other necessary input parameters such as mud resistivity $R_{m}=0.8 \Omega \mathrm{m}$ and borehole radius $r_{h}=0.1016 \mathrm{~m}$.

Fig.3(b) draw the inversed true-formation resistivity $R_{t}$. It indicated that the apparent resistivity of HRDI measurement was less than true-formation resistivity obviously due to the low resistivity in the invasion zone, thus the less apparent resistivity of lateralog 8 device was obtained, as shown in the dash line of Fig.3(a). This low-resistivity invasion zone reduced the readings of HRDI measurement; hence, inversion technique is necessary in order to obtain the true resistivity of formation.

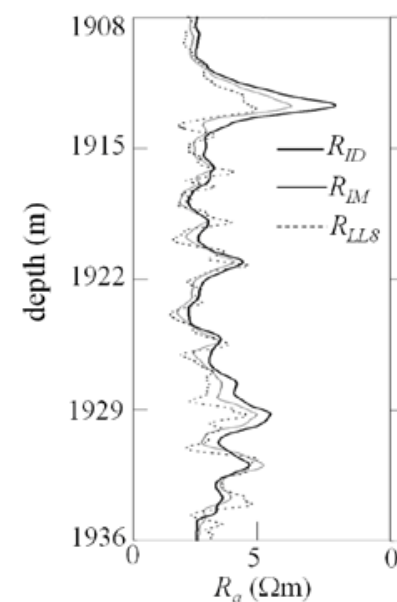

(a)

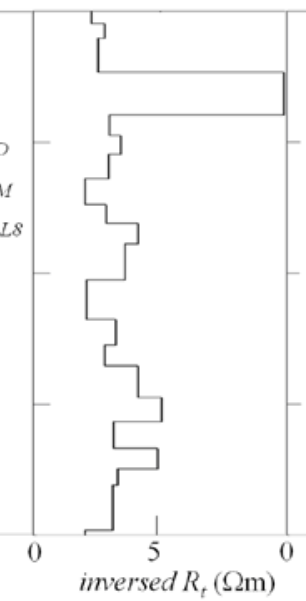

(b)

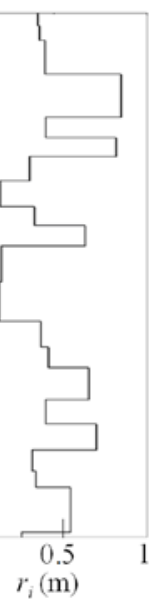

(c)

Figure 3 Logging curves of field HRDI tool and inversion results

(a) HRDI and LL8 logging curves;

(b) inversed true-formation resistivity;

(c) inversed invasion depth

For example, in the interval 1911.2 1913.6m, the present inversion suggested that the formation resistivity was $11.8 \Omega \mathrm{m}$; whereas the HRDI measurement gave $9.36 \Omega \mathrm{m}$ for deep-induction array and $7.36 \Omega \mathrm{m}$ for medium-induction array. Both of them were less than the true resistivity, especially for the medium-induction array. The reason was that the invasion depth became greater in this interval.

Fig. 3(c) illustrated the inversed invasion depth $r_{i}$. In this interval of 1925 1936m, the invasion depth was about $0.5 \mathrm{~m}$. However, in the interval $1911.2 \sim 1913.6 \mathrm{~m}$, the present inversion suggested that the invasion depth was $0.83 \mathrm{~m}$. Great value of invasion depth caused the apparent resistivities of HRDI measurement less than the true value of the formation obviously.

\section{Conclusions}

High-resolution dual induction (HRDI) tools can improve the vertical resolution. Generally, the HRDI measurement can address the thin bed such as the thickness about $0.5 \mathrm{~m}$. The high-resolution medium induction response was sensitive to the resistivity distribution in the vicinity of borehole, whereas the deep induction reading recorded more information of the original formation.

The present study indicated that the inversion algorithm for HRDI measurement is fast and efficient using damping least square method. It can inverse the true-formation resistivity and the invasion depth simultaneously.

A synthetic formation model was constructed to prove the validity of the present algorithm. For thick beds (the thickness $H>1 \mathrm{~m}$ ), the inversed parameters were in good agreement with the model; and for thin beds $(H<1 \mathrm{~m})$, inversed results deviated the true values due to the shoulder effect. 
The application of the present inversion algorithm for a field data indicated that both the trueformation resistivity and the invasion depth can be obtained simultaneously. The inversion can correct the resistivity deviation from the true value due to environmental influences, especially the invasion effect. The inversion results are helpful for log analysts to determine the true-formation resistivity, to estimate the saturation of hydrocarbon, and then to evaluate the reservoir.

\section{Acknowledgment}

The authors would like to thank Prof. Z. H. Liu for her helpful discussions during the inversion calculation

\section{References}

[1] R. M. Bateman and C.E. Lonen, "Log analyst and the programmable pocket calculator - 4. dual induction-laterolog 8”, Log Analyst, vol.19(3), pp.3-7, 1978

[2] L. S. Sinclair and R. W. Strickland, "Coil array for a high resolution induction logging tool and method of logging an earth formation”, U S Patent, 5065099, 1991.

[3] J. Wu, H. S. Gong, J. F. Pang and T. Lu, "A new signal processing method for the dual induction measurement”, Well Logging Technology (in Chinese), vol.27(1), pp.11-15, 2003.

[4] L.Q.Yong, F. Deng, S. M. Zhang, "Iterative method for a class of nonnegative linear least squares problems", Proceedings of the 2010 IEEE International Conference on Progress in Informatics and Computing, PIC 2010, vol.2, pp.1126-1129, 2010

[5] M. Arioli and S. Gratton, "Linear regression models, least-squares problems, normal equations, and stopping criteria for the conjugate gradient method", Computer Physics Communications, vol.183(11), pp.2322-2336, 2012

[6] Damm Tobias and Stahl Dominik, "Linear least squares problems with additional constraints and an application to scattered data approximation”, Linear Algebra and Its Applications, vol.439(4), pp.933-943, 2013 